\title{
Gottron papules: a pathognomonic sign of dermatomyositis
}

\section{Federica Ricceri MD, Francesca Prignano MD PhD}

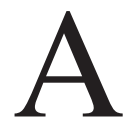
30-year-old woman with a history of psoriasis on her scalp was referred to our dermatology clinic for psoriasis on her hands that had lasted for 1 year and was resistant to topical treatments. The patient had no history of muscle weakness.

On examination, multiple hyperkeratotic, flat, red papules with central atrophy were present on the backs of the patient's metacarpophalangeal and interphalangeal joints (Figure 1). Cuticular hypertrophy and periungual capillary dilatation were also seen (Figure 1, inset). In addition, she had a very subtle heliotrope rash around her eyes. A biopsy of skin from the patient's hand showed acanthosis, hyperkeratosis, focal vacuolar alteration of the basal cell layer, and perivascular inflammatory infiltrate, consistent with Gottron papules. Laboratory investigations

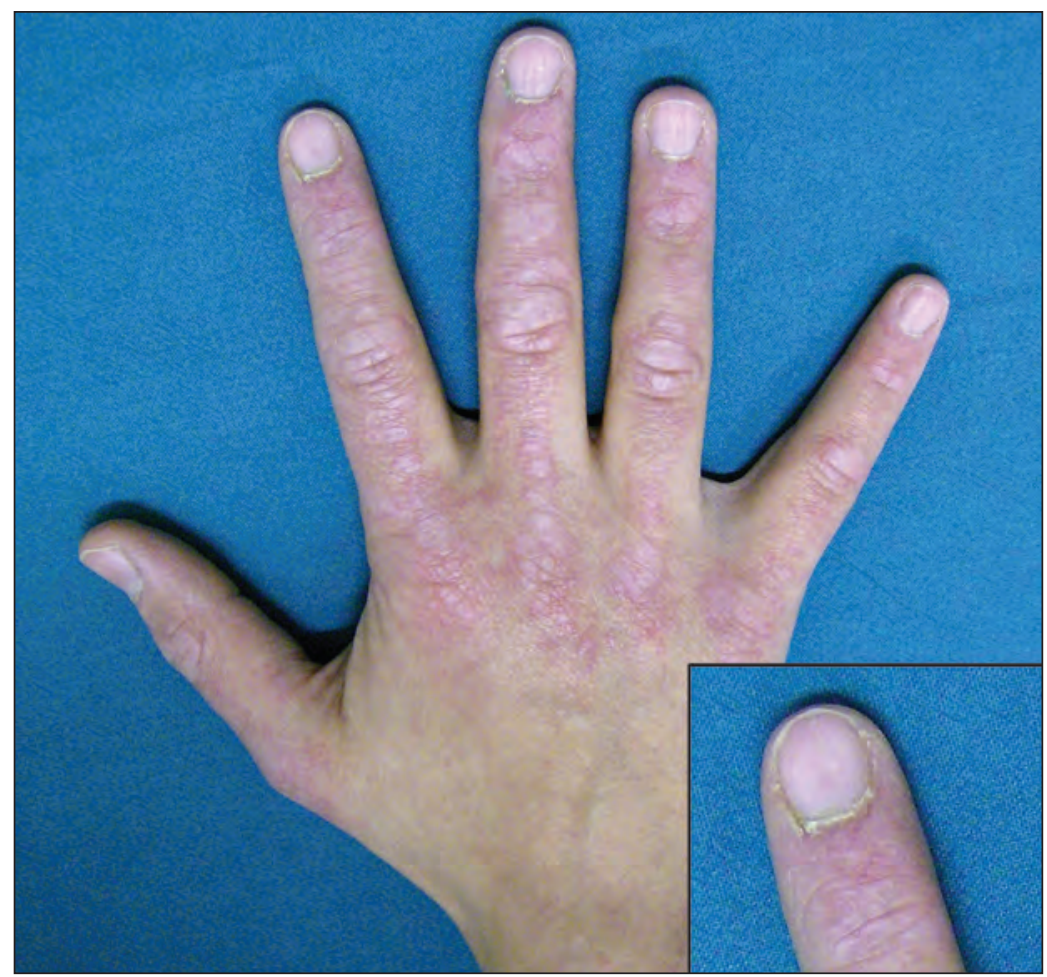

Figure 1: Grotton papules on the hand of a 30-year-old woman. Inset: close-up image of the patient's right index finger showing cuticular hypertrophy and periungual capillary dilatation. showed that the serum levels of muscle enzymes were normal. Electromyography of the proximal muscles (i.e., those close to the trunk) was also normal.

These skin findings in the absence of functional myopathy are characteristic of amyopathic dermatomyositis. We prescribed topical steroids and recommended avoiding exposure to sunlight. The patient's condition showed good clinical improvement.

Dermatomyositis is an uncommon idiopathic inflammatory disorder of the skin and skeletal muscle that affects 10 adults per million and 3.2 children per million worldwide. ${ }^{1}$ Cutaneous manifestations are grouped as pathognomonic, characteristic or compatible. ${ }^{2}$ The pathognomonic features, such as we saw in our patient, include small purple or red flat papules on extensor surfaces, particularly the elbows and joints of the hand (Gottron papules). Although the diagnosis is clinical (Gottron papules are rarely sampled for histopathologic assessment), a punch biopsy is helpful in differentiating the condition from other papulosquamous diseases (e.g., lichen planus, psoriasis), or contact or atopic dermatitis. Changes to the nail fold and a heliotrope rash are included in highly characteristic manifestations. For up to $40 \%$ of patients, skin findings may be the only manifestation. ${ }^{3}$ As in our patient, the absence of muscle disease implies a favourable prognosis.

\section{References}

1. Koler RA, Montemarano A. Dermatomyositis. Am Fam Physician 2001;64:1565-72.

2. Euwer RL, Sontheimer RD. Dermatologic aspects of myositis. Curr Opin Rheumatol 1994;6:583-9.

3. Santmyire-Rosenberger B, Dugan EM Skin involvement in dermatomyositis. Curr Opin Rheumatol 2003;15:714-22.

Competing interests: None declared.

This article has been peer reviewed.

Affiliation: From the Division of Clinical, Preventive and Oncology Dermatology, Department of Critical Care Medicine and Surgery, University of Florence, Florence, Italy

Correspondence to: Francesca Prignano, francesca.prignano @ unifi.it

CMAJ 2013. DOI:10.1503/cmaj.111791 